\title{
ACCION DE NOR-ESTEROIDES EN LA AMENAZA DE ABORTO
}

\author{
Doctores Edmundo G. Murray e Irma J. Rúa
}

La frecuencia del aborto espontáneo sólo puede apreciarse de un modo aproximado, ya que muchos escapan a la estadística. En general se admite una cifra media de un $10 \%$ de todos los embarazos (9).

El tratamiento hormonal de la amenaza de aborto rinde sus mejores resultados cuando existen deficiencias endocrinas apreciables que pueden ser responsables del accidente. El mantenimiento normal de la gestación depende de numerosos factores; entre ellos la presencia de una adecuada actividad progestacional suplida por hormonas de origen endógeno.

Existen evidencias clínicas de que la mayoría de los abortos se acompañan de deficiencia de progesterona, tal como ha sido demostrado por la reducción del pregnanodiol excretado. Es sugestivo, sin embargo, el hecho de que la medicación con progesterona no ha logrado modificar apreciablemente tales circunstancias, reteniendo el embarazo en cifras que no exceden generalmente la mitad de los casos tratados.

Una anidación deficiente del huevo y las anomalías placentarias que de ello se derivan se consideran la consecuencia de transformación deficiente del endometrio.

El hipodesarrollo pregrávido del endometrio se debe frecuentemente a una función deficiente de los ovarios y en esepcial del cuerpo amarillo.

El mecanismo de acción de una hormonoterapia consiste, durante las primeras semanas, en mejorar las condiciones de vida del fruto. Se favorece la formación decidual y se estimula el desarrollo uterino.

Los resultados estadísticos de las medicaciones hormonales son variables. Cuando el fruto es expulsado a las 48 horas siguientes de comenzada la medicación hormonal, puede admitirse que la interrupción del embarazo se ha producido con anterioridad.

El tratamiento instituído en forma temprana, que no se interrumpa prematuramente, conduce en el 70 al $80 \%$ de los casos a la conservación de la gestación $(1,4,6)$.

\section{MATERIAL Y METODO}

Desde 1959 hasta la fecha hemos observado 168 pacientes con síntomas subjetivos, clínicos y de laboratorio, de 
amenaza de aborto, pertenecientes al Servicio de Ginecología del Hospital Juan A. Fernández, a cargo de uno de nosotros.

Todas las pacientes se estudiaron sistemáticamente con los siguientes métodos: observación clínica de los caracteres del dolor y de la metrorragia, modificación de la temperatura de base, centaria, tiroidea y posiblemente suprarrenal, a las cuales puede atribuírse la causa de la amenaza de aborto.

1. Temperatura de base: fue tomada en forma ininterrumpida por todas las pacientes.

2. Colpocitología: se efectuó a diario en los casos en que la metro-

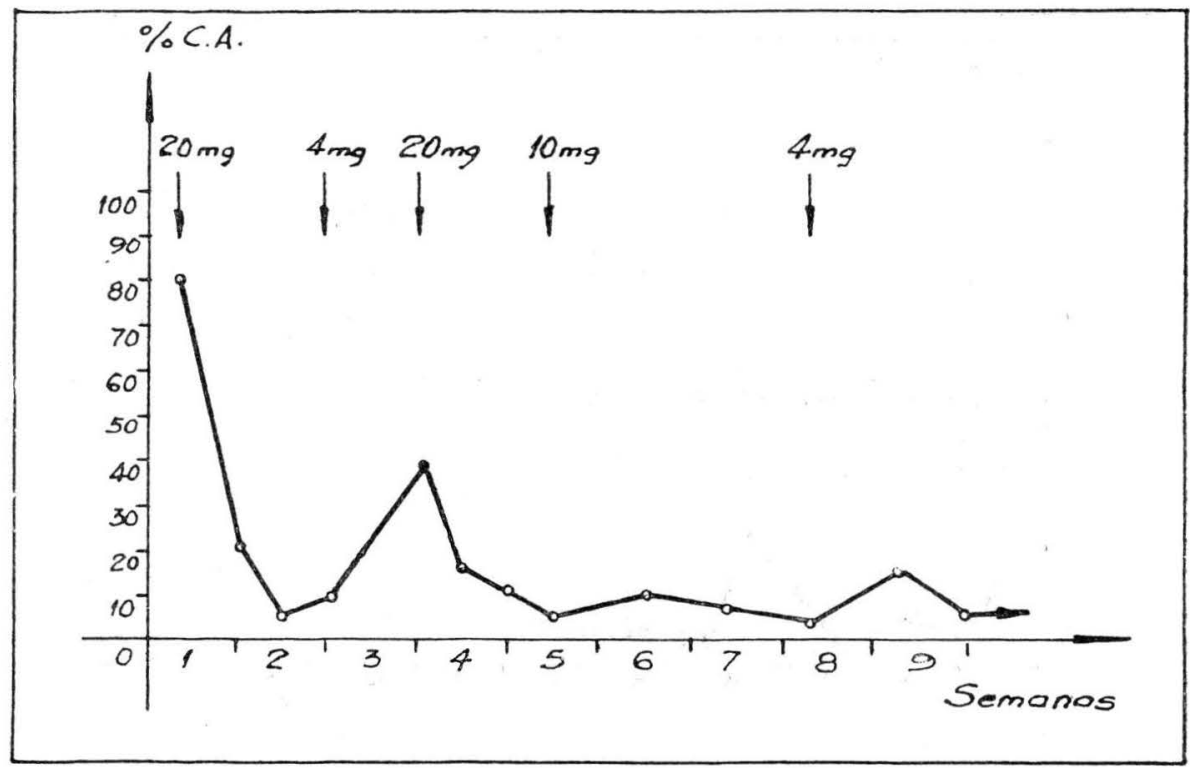

Cuadro 1. Amenaza de aborto. Variación en los porcentajes de células acidófilas con diferentes dosis de nor-esteroides.

variaciones de la colpocitología y urocitograma, modificaciones reológicas y cristalización de la secreción cervical. Los dosajes repetidos del pregnanodiol urinario, 17-cetosteroides y del yodo proteico, han sido elementos valiosos para la orientación terapéutica; de tal manera se ha hallado alteraciones endocrinas relacionadas con la función pla- rragia lo permitía, aumentándose el intervalo al ceder los síntomas de amenaza.

3. Urocitograma: fueron $\mathrm{h}$ e $\mathrm{ch}$ o s sistemáticamente en todos los casos de metrorragias.

4. Secreción cervical: simultáneamente con los extendidos vagi- 
nales se efectuó la prueba de cristalización del mucus, no siendo de mayor utilidad los restantes caracteres del mismo.

5. Observación del dolor: el dolor de tipo congestivo en unas y espasmódico en otras, estuvo presente en todas las pacientes coincidiendo con los demás síntomas de amenaza. el primer trimestre (5 a 15 mgs). Alteraciones en las cifras normales se han hallado en algunos casos con el dosaje del yodo proteico en sangre. El estudio sistemático de los 17-cetosteroides en la amenaza de aborto ha revelado en algunos casos cifras elevadas en su eliminación urinaria.

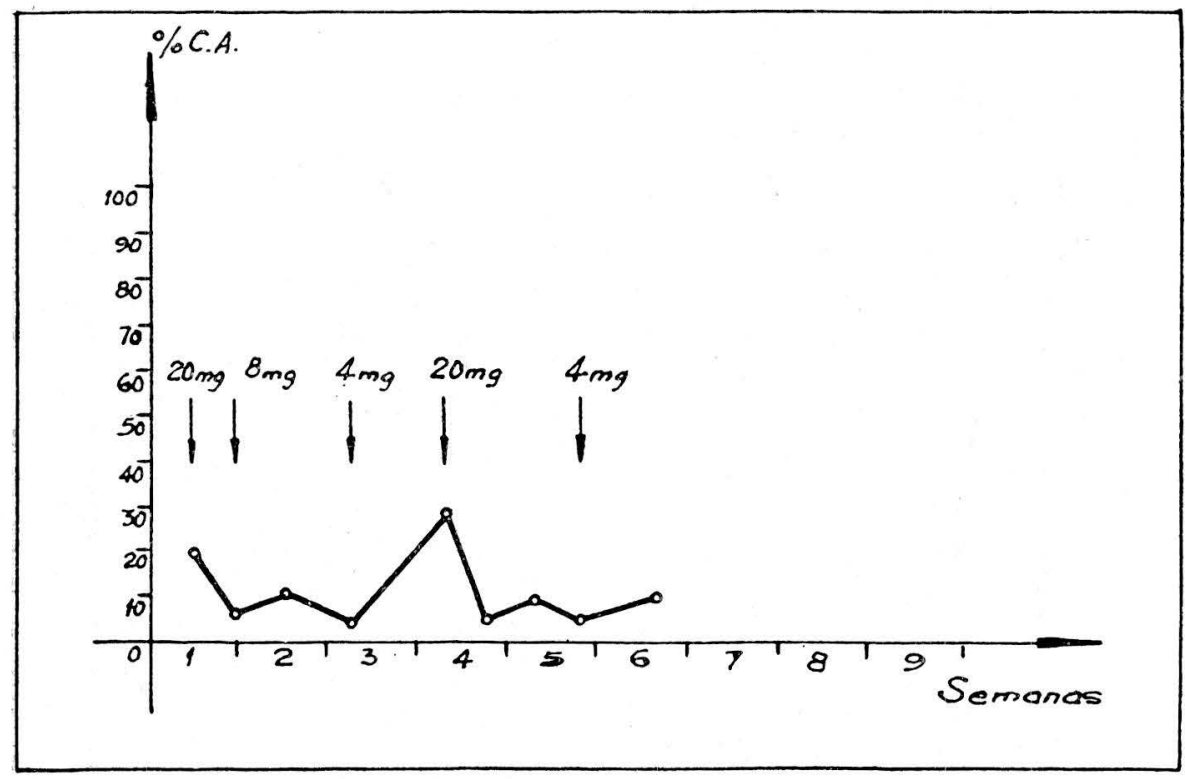

Cuadro 2. Amenaza de aborto. Variación en los porcentajes de células acidófilas con diferentes dosis de nor-esteroides.

6. Observación de metrorragias: de mediana intensidad en algunas y escasas en otras, las profusas se presentaron en muy pocas pacientes.

7. Los dosajes de pregnanodiol urinario con técnica colorimétrica arrojaron valores por debajo de los considerados normales en

\section{TRATAMIENTO Y RESULTADOS}

Solamente consideramos los casos tratados en forma exclusiva con nor-esteroides. Nuestra experiencia con algunas de estas sustancias es aún reducida; mencionaremos en esos casos los resultados sin aportar conclusiones. 
En 120 pacientes se utilizó el acetato de anhidro-hidroxi-nor-progesterona.

En 15, la 17-alfa-vinil-19-nor-testosterona.

En 15, el alilestrenol.

En 8, el noretinodrel.

En un grupo muy reducido, que comprende en total 10 pacientes se utilizó el acetato de 6 alfa-metil-17-alfa-hidroxi-progesterona y la metil-nortestosterona.

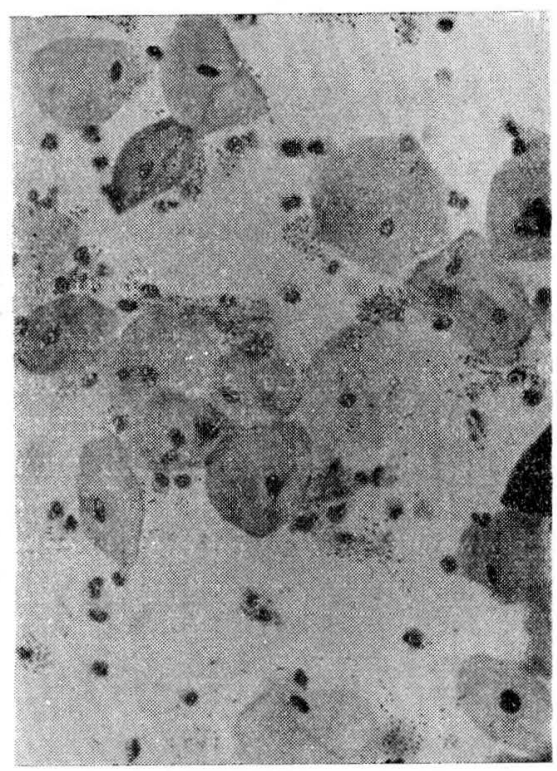

Figura 1. Extendido vaginal. (Técnica de Shörr). Trófico ++ .

Se prescindió de toda otra medicación, particularmente de aquellas de orden antiespasmódico y, sobre todo, tratamientos hormonales asociados.

Entre los medios de observación mencionados anteriormente se descartó la utilidad de la temperatura de base, dado el carácter eminentemente termogénico de los esteroides progestacionales.

Las variaciones citológicas (colpocitología y urocitograma) han demostrado ser el método más eficaz y sencillo para controlar las modificaciones determinadas por la medicación.

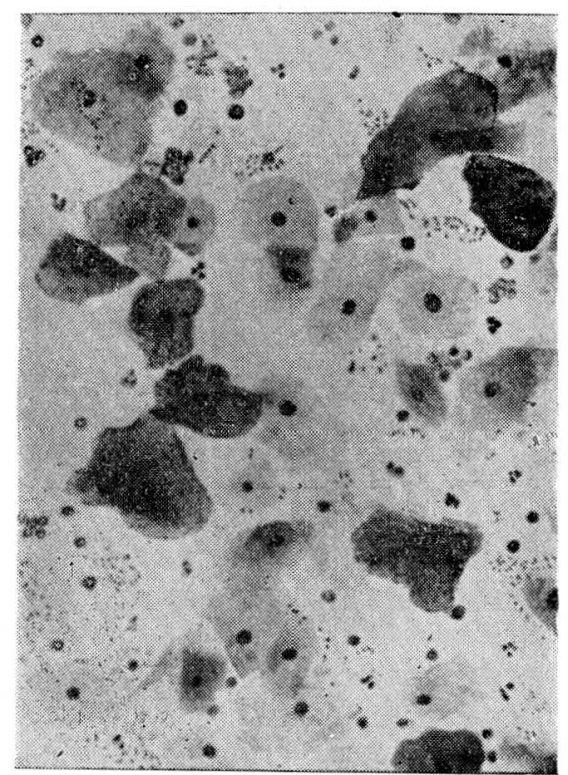

Figura 2. Extendido vaginal. (Técnica de Shörr). Trófico + .

Dosajes semanales del pregnanodiol urinario certificaban la evolución normal o patológica del embarazo, confirmada también por reacciones biológicas.

Para el acetato de anhidro-hidroxinor-progesterona se ensayaron dosis de $4,8,10,16$ y $20 \mathrm{mg}$ en calidad de dosis inicial. Después de estos ensayos se consideró como dosis inicial útil la de $20 \mathrm{mg}$. 
Las dosis de mantenimiento durante las 3 primeras semanas del tratamiento demostraron ser ligeramente más elevadas que las correspondientes a las posteriores.

Dichas dosis deben conservar cierta relación con el grado de eosinofilia colpocitológica existente.

Desde la cuarta semana, en cambio, la dosis de mantenimiento útil no excede de $4 \mathrm{mg}$ diarios.

$\mathrm{Si}$ bien los exámenes citológicos demostraron ser la guía más práctica para el seguimiento de la embarazada portadora de amenaza de aborto endocrina, no es despreciable el valor de los dosajes repetidos del pregnanodiol urinario. Su determinación permite orientar una terapéutica adecuada cuando los valores hallados no corresponden a la edad de la gestación; la importancia de este dato aumenta en aquellas pacientes en cuyas historias cuenta el antecedente de abortos reiterados (7).

Sin poder dar resultados estadísticos los valores del yodo proteico en sangre han sido inferiores a las cifras normales en la amenaza de aborto (5).

De las 120 pacientes tratadas, 85 evolucionaron en forma satisfactoria, habiendo desaparecido en ellas todo peligro de interrupción espontánea (10).

De las 15 pacientes tratadas con la 17-alfa-vinil-19-nor-testosterona, 10 llevaron una evolución favorable del embarazo. Los controles efectuados a este grupo de pacientes son los mismos mencionados para el grupo anterior. Las dosis utilizadas han sido de $15 \mathrm{mg} /$ día durante 5 días, seguidos de $10 \mathrm{mg} /$ día durante otros 5 días, para continuar con $5 \mathrm{mg} /$ día como dosis de sostén.
La severidad de la hemorragia y/o dolores es reducida en pocas horas y dominada en forma marcada o completa entre las 12 y 24 horas.

El alilestrenol ha sido descrito por Majderek (8) "como un gestágeno oral que mantiene el embarazo".

Hasta ahora tenemos muy buenos informes sobre el alilestrenol (12). Una de las investigaciones más recientes es la de Borglin (2) que comprende 97 casos de aborto habitual o inminente, y en la que en 59 casos un tratamiento con alilestrenol a la dosis de 5 a $30 \mathrm{mg}$ al día mantuvo el embarazo hasta que el feto fue viable.

En la actualidad trabajamos activamente con esta medicación. Los resulta-

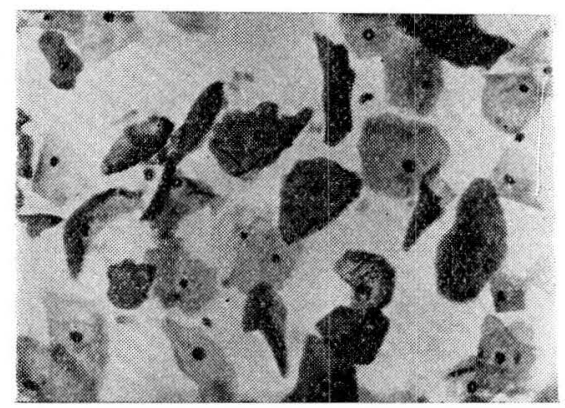

Figura 3. Extendido vaginal. (Técnica de Shörr). Hipotrófico + .

dos obtenidos hasta ahora no son tan halagadores; pacientes con antecedentes de abortos reiterados y con signos y síntomas de una nueva amenaza de origen endocrino debieron ser medicadas con una terapéutica asociada (estrógenos), ya que el cuadro no cedía frente a la indicación aislada del alilestrenol en dosis de $15 \mathrm{mg} /$ día. 
En trabajos posteriores mencionaremos resultados definitivos.

El noretinodrel lo hemos utilizado en un pequeño número de pacientes, más que como medicación terapéutica, ha sido empleado como profiláctico en los casos de aborto habitual y tan pronto ha sido diagnosticado un nuevo embarazo. Los contados casos tratados nos han dado resultados favorables que coinciden con estadísticas mayores (11) que indican la evolución a término de esos embarazos sin registrar ninguna anormalidad en los niños nacidos de madres tratadas con 20 mgs de noretinodrel, dosis empleadas por nosotros.

En un grupo reducido de 10 pacientes se utilizaron los derivados metílicos de la testosterona. El conocimiento de los efectos virilizantes y androgenizantes de estas sustancias relatadas en experiencias clínicas y animales por otros autores (3) (13) nos ha llevado a la utilización de dosis bajas, que consideramos insuficientes, y por ello a la obtención de resultados poco favorables, no dignos de señalar y que le restan todo valor estadístico.

Ninguno de los nor-esteroides utilizados han mostrado efectos secundarios desagradables. No fueron observados signos de virilización en las madres así tratadas ni signos de masculinización en los recién nacidos. El total de estas niñas ha sido examinado meticulosamente, no habiéndose comprobado hasta la fecha ninguna alteración imputable al uso de los esteroides empleados.

\section{RESUMEN Y CONCLUSIONES}

1. Se observaron 168 pacientes con síntomas subjetivos, clínicos y de laboratorio, de amenaza de aborto pertenecientes al Servicio de Ginecología del Hospital Juan A. Fernández a cargo de uno de nosotros.

2. Fueron métodos de estudio: temperatura de base, colpocitología, urocitograma, secreción cervical y determinaciones hormonales (pregnanodiol urinario, yodo proteico en sangre y 17cetosteroides).

3. Los nor-esteroides utilizados han sido:

Acetato de anhidro-hidroxi-norprogesterona - 120 pacientes.

17-alfa-vinil-19-nor-testosterona - 15 pacientes.

Alilestrenol - 15 pacientes.

Noretinodrel - 8 pacientes.

Acetato de 6 alfa-metil-17-alfahidroxiprogesterona y metil-nortestosterona - 10 pacientes.

4. Las variaciones citológicas (colpocitología y urocitograma) han demostrado ser el método más eficaz y sencillo para controlar la evolución del embarazo, confirmada por dosajes repetidos de pregnanodiol urinario.

5. Las dosis iniciales y de mantenimiento y los resultados obtenidos se resumen a continuación en la siguiente tabla:

6. Los mejores resultados fueron obtenidos con el acetato de an- 
hidro-hidroxi-nor-progesterona y la 17-alfa-vinil-19-nor-testosterona.

7. Dosis relativamente elevadas no produjeron efectos colaterales secundarios.

8. Ninguno de los nor-esteroides utilizados llevó a la aparición de
Rev. Col. Obs. y Ginec.

signos de virilización en las madres así tratadas ni signos de masculinización en las recién nacidas.

9. Constituye este trabajo una evaluación crítica de la eficacia de algunos nor-esteroides en el tratamiento de la amenaza de aborto de origen endocrino.

\begin{tabular}{|c|c|c|c|c|c|}
\hline NOR-ESTEROIDE & $\begin{array}{l}\text { Dosis } \\
\text { inicial }\end{array}$ & $\begin{array}{l}\text { Wosis mante- } \\
\text { nimiento }\end{array}$ & $\begin{array}{l}\text { Núm. } \\
\text { de pa- } \\
\text { cientes }\end{array}$ & $\begin{array}{l}\text { Evolución } \\
\text { favorable }\end{array}$ & $\begin{array}{l}\text { Porcen- } \\
\text { taje }\end{array}$ \\
\hline Acetato de anhidro-hidroxi-norprogesterona & $20 \mathrm{mg}$. & $4 \mathrm{mg}$. & 120 & 85 & $70,83 \%$ \\
\hline 17-alfa-vinil-19-nortestosterona $\ldots$. . . . . & $15 \mathrm{mg}$ & $5 \mathrm{mg}$. & 15 & 10 & $66,66 \%$ \\
\hline 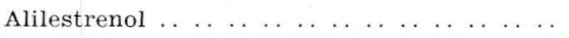 & $15 \mathrm{mg}$ & $15 \mathrm{mg}$. & 15 & 8 & $53,33 \%$ \\
\hline 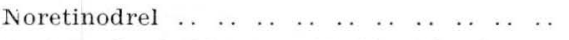 & $20 \mathrm{mg}$. & $10 \mathrm{mg}$ & 8 & 5 & - \\
\hline $\begin{array}{l}\text { Acetato de } 6 \text { alfa-metil-17-alfa-hidroxi-pro- } \\
\text { gesterona y metil-nortestosterona ... . }\end{array}$ & $10 \mathrm{mg}$. & $5 \mathrm{mg}$ & 10 & - & - \\
\hline
\end{tabular}

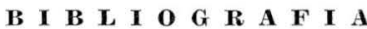

1. BISHOP, P. M. F.: Brit. Med. J., 4671, 130, 1950.

2. Borglin, N. E.: Acta Endocr. Suppl. 58: 1960.

3. BUR, G. E., y MONTUORI, E.: Bol. Soc. Obst. y Ginec. de Bs. As. 38: 536, 1959.

4. Castelazo Ayala, L.: Ginec. Obst. Méx. 8: 167, 1953.

5. ENRIORI, C. L.: Determinación del yodo proteico (en preparación).

6. GITSCH, E. : Wien. Klin. Wschr. 68: 370, 1956.

7. LANCMAde, Ch. F., NOtrica, S., Demetrion Ph. D., y WARE, A. G.: Am. J. Obst. and Gynee. 81: 1149, 1961.

8. MAJDEREK, Z., J. de VISSER, J. van der VIES, y OVERBEEK, G. A.: Acta endocr. 35: 8, 1960 .

9. MURray, E. G. y RUA, I. J. : 9o Congr. Chileno de Obst. y Ginec. (en prensa) 1961.

10. murray, E. G., RUA, I. J., RAmella, J. E., y Simone, L. H.: XI Congr. Argentino de Obst. y Ginec. $13 \%$ sesión plenaria. Octubre 1961.

11. Rakoff, A. E.: Ann. New York Acad. Se. 71: 484, 1958.

12. TAUSK, M.: Coloquio sobre Nor-esteroides progestacionales. 1 Vol. pág. 33, edit. Centro de Endocrinología, Buenos Aires, 1961. 